\title{
Fuzzy decision making in complex frameworks with generalized aggregation operators
}

José M. Merigó ${ }^{1,2^{*}}$, Anna M. Gil-Lafuente ${ }^{3}$, Dejian $\mathrm{Yu}^{4}$, Carlos Llopis-Albert ${ }^{5}$

${ }^{1}$ Manchester Business School, University of Manchester Booth Street West, M15 6PB Manchester, UK

${ }^{2}$ Department of Management Control and Information Systems, School of Economics and Business, University of Chile, Av. Diagonal Paraguay 257, 8330015 Santiago, Chile

${ }^{3}$ Department of Business Administration, University of Barcelona Av. Diagonal 690, 08034 Barcelona, Spain

${ }^{4}$ School of Information, Zhejiang University of Finance and Economics,

18 Xueyuan Street, Hangzhou, 310018, China

${ }^{5}$ Instituto Geológico y Minero de España (IGME),

c/Cirilo Amorós, 42 - Entreplanta, 46004 Valencia, Spain

${ }^{*}$ Corresponding author: Tel: +34 934021962; Fax: +34 934039882.

Email address: J.M. Merigó (jmerigo@fen.uchile.cl), A.M. Gil (amgil@ub.edu), D. Yu

(yudejian@126.com).C. Llopis (cllopisa@gmail.com).

\section{Graphical abstract}

Families of FGUAO operators.

\begin{tabular}{|c|c|c|c|c|c|c|c|c|}
\hline & $\lambda=$ & $\ldots$ & $\lambda=-1$ & $\lambda \rightarrow 0$ & $\lambda=1$ & $\lambda=2$ & $\ldots$ & $\lambda=$ \\
\hline$\delta$ & Min & $\ldots$ & Min & Min & Min & Min & $\ldots$ & Max \\
$-\infty$ & & & & & & & & \\
\hline$\ldots$ & $\ldots$ & $\ldots$ & $\ldots$ & $\ldots$ & $\ldots$ & $\ldots$ & $\ldots$ & $\ldots$ \\
\hline$\delta$ & Min & $\ldots$ & FHUHAO & FHUGAO & FHUAO & FHUQAO & $\ldots$ & Max \\
$=$ & & & & & & & & \\
-1 & & & & & & & & \\
\hline$\delta$ & Min & $\ldots$ & FGUHAO & FGUGAO & FGUAO $*$ & FGUQAO & $\ldots$ & Max \\
$\rightarrow$ & & & & & & & & \\
0 & & & & & & & & \\
\hline$\delta$ & Min & $\ldots$ & FUHAO & FUGAO & FUAO & FUQAO & $\ldots$ & Max \\
$=$ & & & & & & & & \\
\hline
\end{tabular}




\begin{tabular}{|c|c|c|c|c|c|c|c|c|}
\hline $\begin{array}{c}1 \\
\delta\end{array}$ & Min & $\ldots$ & FQUHAO & FQUGAO & FQUAO & FQUQAO & $\ldots$ & Max \\
2 & & & & & & & & \\
\hline$\ldots$ & $\ldots$ & $\cdots$ & $\ldots$ & $\ldots$ & $\ldots$ & $\ldots$ & $\ldots$ & $\ldots$ \\
\hline $\begin{array}{c}\delta \\
=\end{array}$ & Min & $\ldots$ & $\operatorname{Max}$ & $\operatorname{Max}$ & $\operatorname{Max}$ & $\operatorname{Max}$ & $\ldots$ & Max \\
$\infty$ & & & & & & & & \\
\hline
\end{tabular}

\title{
Research highlights
}

- A new approach for fuzzy decision making with complex environments.

- The fuzzy generalized unified aggregation operator.

- A wide range of new fuzzy aggregation operators.

- An application in a fuzzy economic multi-person decision making.

- New methods for decision making in macroeconomics.

\begin{abstract}
This article presents a new aggregation system applied to fuzzy decision making. The fuzzy generalized unified aggregation operator (FGUAO) is a system that integrates many operators by adding a new aggregation process that considers the relevance that each operator has in the analysis. It also deals with an uncertain environment where the information is studied with fuzzy numbers. A wide range of particular cases and properties are studied. This approach is further extended by using quasi-arithmetic means. The paper ends studying the applicability in decision making problems regarding the European Union decisions. For doing so, the work uses a multi-person aggregation process obtaining the multi-person - FGUAO operator. An example concerning the fixation of the interest rate by the European Central Bank is presented.
\end{abstract}

Keywords: OWA operator; Fuzzy numbers; Aggregation operators; Group decision-making. 


\section{Introduction}

Aggregation operators are very useful in many areas since they collect data and provide some general results of the available information. Averaging aggregation operators are very common including the arithmetic mean, the weighted average, the probabilistic aggregation and the ordered weighted average (OWA) $[1,10,40]$. Sometimes, the data is imprecise and cannot be studied with exact numbers, we need to use other tools such as the fuzzy numbers [43-44] that can be implemented following the extension principle [44]. The key benefit is that they represent the imprecise information considering the minimum, the maximum, the most expected results and the possibility between 0 and 1 that the internal values of the interval will occur [6,12]. Many extensions of the crisp aggregation operators into a fuzzy environment are available in the literature [5,16,30-31].

Another framework for representing the data in a more general way when dealing with these operators is the use of generalized and quasi-arithmetic means [9,37]. The key advantage is that they consider many particular cases including quadratic, geometric, arithmetic and harmonic aggregations. Wang and Luo [29] developed a fuzzy version of the weighted quasi-arithmetic mean. Merigó and Casanovas [21] introduced the fuzzy generalized OWA operator and Merigó and Gil-Lafuente [23] extended this approach by using induced aggregation operators and linguistic information. Zhao et al. [48] studied the use of intuitionistic fuzzy sets and Zeng et al. [47] an approach based on distance measures. Zeng and $\mathrm{Su}$ [46] also studied intuitionistic fuzzy sets with distance measures. Liu et al. [15] considered the use of generalized means in continuous aggregations and Zhou et al. [49] with power averages. 
When analyzing the aggregation operators explained in the previous paragraphs, usually, they are treated in a separate way. However, it would be useful to be able to represent some of them in a unified framework in order to assess complex environments with various sources of information that have to be analyzed with different aggregation operators. Several attempts are found in the literature such as the immediate probability $[7,18,38]$ that integrates the probability with the OWA operator in the same aggregation, the hybrid average [32], the weighted OWA operator [28] and the importance OWA [30] that integrate the weighted average with the OWA operator. Recently, Merigó has suggested several new extensions that consider the degree of importance that each concept has in the analysis such as the OWA weighted average [25], the probabilistic OWA [19] and the probabilistic weighted average $[20]$.

The aim of this paper is to introduce a new approach for dealing with complex frameworks where the available data is imprecise and can be assessed with fuzzy numbers. We present the fuzzy generalized unified aggregation operator (FGUAO). The FGUAO is an aggregation operator that provides a unified system of fuzzy aggregation operators by adding a new aggregation that includes all the particular aggregations of an aggregation system considering the importance that each sub aggregation has in the analysis. The main benefit of this generalization is that we can visualize complex environments in a more flexible way because we can analyze different perspectives of the same problem considering different sources of information. Thus, it permits us to study the information by using a more general perspective that avoids the loss of information. Moreover, it uses generalized aggregation operators $[9,37]$ in order to obtain a more general representation by using generalized and quasi-arithmetic means. Therefore, it includes the majority of fuzzy averaging aggregation operators as particular cases. 
Some key properties of the FGUAO operator are studied including several particular cases. The FGUAO includes many operators including the fuzzy UAO, fuzzy generalized weighted average and the fuzzy generalized OWA operator. The work also considers situations where the generalized aggregation operators become arithmetic, geometric or quadratic including the quadratic FUAO and the geometric FUAO operator. A further extension to this approach is introduced with quasi-arithmetic means building the fuzzy quasiarithmetic UAO (Quasi-FUAO) operator. This operator generalizes the FGUAO operator as a particular formulation by using a strictly continuous monotonic function.

We also study its applicability focusing on a decision making problem $[3,41]$ regarding the European Union decisions. Note that this type of situations represent cases of decision making in macroeconomics which usually involve huge quantities of money and small mistakes may produce huge loses. Therefore, complete and very well-assessed decision making models are needed. In this paper, we use a multi-person aggregation system that forms the multi-person - FGUAO operator. An illustrative example in multi-criteria group decision making is presented. The example is focused on the fixation of the general interest rate for the Eurozone by the European Central Bank. Observe that small decimal variations may produce huge differences in the economic structure of the region.

The paper is organized as follows. Section 2 presents the FGUAO operator and Section 3 some families of FGUAO operators. Section 4 introduces the Quasi-FUAO operator. Section 5 studies its applicability in a multi-person decision making, Section 6 develops an illustrative example and Section 7 shows the main findings of the work. 


\section{The fuzzy generalized unified aggregation operator}

The FGUAO operator is a generalized aggregation operator that unifies many aggregation operators considering their degree of importance in the analysis and in an imprecise environment that requires the use of fuzzy numbers. Moreover, it uses generalized means allowing the aggregation to be arithmetic, quadratic or geometric. By including a wide range of sub aggregation systems, it can adapt to many different scenarios without losing information or avoiding it as much as possible. The FGUAO is defined as:

$\operatorname{FGUAO}\left(\tilde{a}_{1}, \tilde{a}_{2}, \ldots, \tilde{a}_{n}\right)=\sum_{h=1}^{m} C_{h}\left(\sum_{i=1}^{n} w_{i}^{h} \widetilde{a}_{i}^{\lambda_{h}}\right)^{1 / \lambda_{h}}$,

where $C_{h}$ is the relevance that each sub aggregation has in the system with $C_{h} \in[0,1]$ and $\sum_{h=1}^{m} C_{h}=1, w_{i}^{h}$ is the $i$ th weight of the $h$ th weighting vector $W$ with $w_{i}^{h} \in[0,1]$, $\sum_{i=1}^{n} w_{i}^{h}=1, \lambda_{h}$ is a parameter such that $\lambda_{h} \in(-\infty, \infty)$ and $\tilde{a}_{i}$ is the argument variable that uses fuzzy numbers.

Note that in order to make operations on fuzzy numbers in the FGUAO operator, we follow the methodology explained by Dubois and Prade [6] and Kaufmann and Gupta [12]. Observe that a further generalization of the FGUAO operator is possible by assuming that the weighting vector $C$ has to be included in a generalized aggregation process. Then, we get the following expression:

$F G U A O\left(\tilde{a}_{1}, \tilde{a}_{2}, \ldots, \tilde{a}_{n}\right)=\left(\sum_{h=1}^{m} C_{h}\left(\left(\sum_{i=1}^{n} w_{i}^{h} \widetilde{a}_{i}^{\lambda_{h}}\right)^{1 / \lambda_{h}}\right)^{\delta}\right)^{1 / \delta}$, 
where $\delta$ is a parameter such that $\delta \in(-\infty, \infty)$. Note also that we assume that all the $\lambda_{h}$ are usually the same for all the sub aggregations but it is possible to consider for each sub aggregation a different parameter in more complex representations.

Observe that if the arguments that are represented with fuzzy numbers become crisp numbers, the FGUAO operator becomes the unified aggregation operator (UAO).

When dealing with fuzzy numbers $[6,12,24]$, it is worth noting that sometimes it is not clear how to rank them. In these cases, it is necessary to use a method for ranking fuzzy numbers. In the literature, there are a lot of methods [27] but for simplicity let us use the method explained by Merigó [19] focusing on the value available in the highest membership degree $(\alpha=1)$. Note that if this value is an interval, the method considers the average of this 2-tuple. Thus, if we defuzzify a triangular fuzzy number, for example [10, 15, 20], we would assume 15 as the crisp value. And if a trapezoidal fuzzy number is defuzzified such as $[20,30$, $40,50]$, the outcome would be $(30+40) / 2=35$. Moreover, if the weights $w_{i}^{h}$ or the weights $C_{h}$ do not sum up to 1 , it is necessary to normalize the aggregation as follows:

$\operatorname{FGUAO}\left(\tilde{a}_{1}, \tilde{a}_{2}, \ldots, \tilde{a}_{n}\right)=\left(\frac{1}{C_{h}} \sum_{h=1}^{m} C_{h}\left(\left(\frac{1}{W^{h}} \sum_{i=1}^{n} w_{i}^{h} \widetilde{a}_{i}^{\lambda_{h}}\right)^{1 / \lambda_{h}}\right)^{\delta}\right)^{1 / \delta}$

Note that this is common if dealing with weighting vectors that can also be represented with fuzzy numbers. The FGUAO accomplishes properties such as monotonicity, boundedness and idempotency. Observe that idempotency occurs if $\tilde{a}_{i}=\tilde{a}$, for all $i \in\{1,2$, $\ldots, n\}$, then: $f\left(\tilde{a}_{1}, \tilde{a}_{2}, \ldots, \tilde{a}_{n}\right)=\tilde{a}$. The boundary condition indicates that $\operatorname{Min}\left\{a_{i}\right\} \leq f\left(\tilde{a}_{1}, \tilde{a}_{2}\right.$, $\left.\ldots, \tilde{a}_{n}\right) \leq \operatorname{Max}\left\{a_{i}\right\}$. And the monotonicity proves that if $\tilde{a}_{i} \geq \tilde{e}_{i}$, for all $i$, then: $F G U A O\left(\tilde{a}_{1}, \tilde{a}_{2}\right.$, $\left.\ldots, \tilde{a}_{n}\right) \geq F G U A O\left(\tilde{e}_{1}, \tilde{e}_{2}, \ldots, \tilde{e}_{n}\right)$. Note that the proofs of these theorems are trivial and thus omitted. 
Another interesting property of the FGUAO operator is that it also includes many partial bounds by considering the bounds in one of the aggregation operators but not in all of them. Observe that this situation is common in operators such as the probabilistic OWA [19], the probabilistic weighted average [20], the OWA weighted average [24], the probabilistic OWA weighted average [25], the immediate probability, the weighted OWA and the hybrid average. From a general point of view, a semi-bound appears when at least one of the sub aggregations is bounded by the minimum and the maximum.

Theorem 1 (Semi boundary condition). Assume $f$ is the FGUAO operator, then:

$$
\begin{aligned}
& C_{1} \times \operatorname{Min}\left\{\tilde{a}_{i}\right\}+\sum_{h=2}^{m} C_{h}\left(\sum_{i=1}^{n} w_{i}^{h} \widetilde{a}_{i}^{\lambda_{h}}\right)^{1 / \lambda_{h}} \leq f\left(\tilde{a}_{1}, \tilde{a}_{2}, \ldots, \tilde{a}_{n}\right) \leq \mathrm{C}_{1} \times \operatorname{Max}\left\{\tilde{a}_{i}\right\}+ \\
& \sum_{h=2}^{m} C_{h}\left(\sum_{i=1}^{n} w_{i}^{h} \widetilde{a}_{i}^{\lambda_{h}}\right)^{1 / \lambda_{h}} . \text { (4) }
\end{aligned}
$$

Proof. It is trivial and thus omitted.

Moreover, it is interesting to characterize the weights of the FGUAO operator. For doing so, we can use for example the entropy of dispersion. In this case, we get the following:

$$
H(W)=\sum_{h=1}^{m} C_{h}\left(-\sum_{i=1}^{n} w_{i} \ln \left(w_{i}\right)\right)
$$

If $C_{h}=1$, the entropy becomes the Shannon entropy [26] for the particular aggregation we are considering. Note that if the particular aggregation operator we consider is an OWA aggregation [39], then, we are analyzing the Yager entropy [33]. Furthermore, observe that it would be feasible to consider a similar analysis in the orness measure, the divergence of the weighting vector and the balance operator $[19,33]$.

\section{Families of FGUAO operators}


The FGUAO includes many particular types of sub aggregations. Thus, a key feature of the FGUAO operator is that it includes a wide range of aggregation operators that can be seen as particular representations of this more general framework. First, let us analyze the fuzzy generalized POWAWA (FGPOWAWA) operator. The FGPOWAWA appears if we simplify the FGUAO operator by using the fuzzy generalized weighted average (FGWA), the fuzzy generalized probabilistic aggregation (FGPA) and the fuzzy generalized OWA (FGOWA) in the same formulation. That is:

FGPOWAWA $\left(\tilde{a}_{1}, \tilde{a}_{2}, \ldots, \tilde{a}_{n}\right)=C_{1} \sum_{j=1}^{n} w_{j}^{1} b_{j}+C_{2} \sum_{i=1}^{n} w_{i}^{2} \widetilde{a}_{i}+C_{3} \sum_{i=1}^{n} w_{i}^{3} \widetilde{a}_{i}$,

where $b_{j}$ is the $j$ th largest of the $\tilde{a}_{n}, w^{1}$ represents the OWA weights, $w^{2}$ the weighted average and $w^{3}$ the probabilities.

Note that when dealing with the OWA operator, an additional ordering is needed in order to aggregate the information as shown in Eq. (9). However, it is also possible to solve this problem by adapting the reordering of the OWA to the initial ordering [36]. As we can see, the FGPOWAWA considers many other particular types of operators such as:

- $C_{1}=1 \rightarrow$ FGOWA.

- $C_{2}=1 \rightarrow$ FGWA.

- $C_{3}=1 \rightarrow$ FGPA.

- $\quad C_{3}=0, \rightarrow$ fuzzy generalized OWAWA (FGOWAWA).

- $C_{2}=0, \rightarrow$ fuzzy generalized probabilistic OWA (FGPOWA).

- $C_{1}=0, \rightarrow$ fuzzy generalized probabilistic weighted average (FGPWA).

By focusing on the generalized structure of the aggregation, we can analyze other particular families studying different results in the parameter $\lambda$ and $\delta$. Observe that the value to be used here depends on the type of problem we want to study. Usually, in the literature it 
has been shown that the arithmetic aggregations are the most practical ones although in some specific situations some other aggregations may become more useful such as in multiplicative preference relations where it is very common to use geometric aggregations. For example:

- If $\lambda_{h}=1$ for all $h$ and $\delta=1$, we build the fuzzy UAO (FUAO) operator.

$\operatorname{FUAO}\left(\tilde{a}_{1}, \tilde{a}_{2}, \ldots, \tilde{a}_{n}\right)=\sum_{h=1}^{m} C_{h} \sum_{i=1}^{n} w_{i}^{h} \widetilde{a}_{i}$

- If $\lambda_{h} \rightarrow 0$ for all $h$ and $\delta=1$, the fuzzy unified geometric aggregation operator (FUGAO) operator:

$\operatorname{FUGAO}\left(\tilde{a}_{1}, \tilde{a}_{2}, \ldots, \tilde{a}_{n}\right)=\sum_{h=1}^{m} C_{h} \prod_{i=1}^{n} \widetilde{a}_{i}^{w_{i}}$

- If $\lambda_{h}=2$ for all $h$ and $\delta=1$, the fuzzy unified quadratic aggregation operator (FUQAO) operator:

$F U Q A O\left(\tilde{a}_{1}, \tilde{a}_{2}, \ldots, \tilde{a}_{n}\right)=\sum_{h=1}^{m} C_{h}\left(\sum_{i=1}^{n} w_{i}^{h} \widetilde{a}_{i}^{2}\right)^{1 / 2}$

- If $\lambda_{h}=-1$ for all $h$ and $\delta=1$, the fuzzy unified harmonic aggregation operator (FUHAO) operator:

$$
\operatorname{FUHAO}\left(\tilde{a}_{1}, \tilde{a}_{2}, \ldots, \tilde{a}_{n}\right)=\sum_{h=1}^{m} C_{h} \frac{1}{\sum_{i=1}^{n} \frac{w_{i}}{\tilde{a}_{i}}} .
$$

- If $\lambda_{h}=3$ for all $h$ and $\delta=1$, the fuzzy unified cubic aggregation operator (FUCAO) operator:

$\operatorname{FUCAO}\left(\tilde{a}_{1}, \tilde{a}_{2}, \ldots, \tilde{a}_{n}\right)=\sum_{h=1}^{m} C_{h}\left(\sum_{i=1}^{n} w_{i}^{h} \widetilde{a}_{i}^{3}\right)^{1 / 3}$

- If $\lambda_{h} \rightarrow 0$ for all $h$ and $\delta \rightarrow 0$, the fuzzy geometric unified geometric aggregation operator (FGUGAO) operator: 
$\operatorname{FGUGAO}\left(\tilde{a}_{1}, \tilde{a}_{2}, \ldots, \tilde{a}_{n}\right)=\prod_{h=1}^{m} C_{h} \prod_{i=1}^{n} \widetilde{a}_{i}^{w_{i}}$

- If $\lambda_{h}=2$ for all $h$ and $\delta=2$, the fuzzy unified quadratic aggregation operator (FQUQAO) operator:

$\operatorname{FQUQAO}\left(\tilde{a}_{1}, \tilde{a}_{2}, \ldots, \tilde{a}_{n}\right)=\sqrt{\sum_{h=1}^{m} C_{h}^{2}\left(\sum_{i=1}^{n} w_{i}^{h} \widetilde{a}_{i}^{2}\right)^{1 / 2}}$.

- If $\lambda_{h}=-1$ for all $h$ and $\delta=-1$, the fuzzy unified harmonic aggregation operator (FHUHAO) operator:

FHUHAO $\left(\tilde{a}_{1}, \tilde{a}_{2}, \ldots, \tilde{a}_{n}\right)=\sum_{h=1}^{m} C_{h} \sum_{i=1}^{n} \frac{w_{i}}{\widetilde{a}_{i}}$

- If $\lambda_{h}=3$ for all $h$ and $\delta=3$, the fuzzy unified cubic aggregation operator (FCUCAO) operator:

$\operatorname{FCUCAO}\left(\tilde{a}_{1}, \tilde{a}_{2}, \ldots, \tilde{a}_{n}\right)=\left(\sum_{h=1}^{m} C_{h}^{3}\left(\sum_{i=1}^{n} w_{i}^{h} \widetilde{a}_{i}^{3}\right)^{1 / 3}\right)^{1 / 3}$

In summary, a first level of particular cases of FGUAO operators can be studied by analysing different values in $\lambda$ and $\delta$ as shown in Table 1 .

Table 1

Types of FGUAO operators.

\begin{tabular}{|c|c|c|c|c|c|c|c|c|}
\hline & $\lambda=-\infty$ & $\ldots$ & $\lambda=-1$ & $\lambda \rightarrow 0$ & $\lambda=1$ & $\lambda=2$ & $\ldots$ & $\lambda=\infty$ \\
\hline$\delta=-\infty$ & Min & $\ldots$ & Min & Min & Min & Min & $\ldots$ & Max \\
\hline$\cdots$ & $\cdots$ & $\cdots$ & $\cdots$ & $\cdots$ & $\cdots$ & $\cdots$ & $\cdots$ & $\cdots$ \\
\hline$\delta=-1$ & Min & $\ldots$ & FHUHAO & FHUGAO & FHUAO & FHUQAO & $\ldots$ & Max \\
\hline$\delta \rightarrow 0$ & Min & $\ldots$ & FGUHAO & FGUGAO & FGUAO* & FGUQAO & $\ldots$ & Max \\
\hline$\delta=1$ & Min & $\ldots$ & FUHAO & FUGAO & FUAO & FUQAO & $\ldots$ & Max \\
\hline
\end{tabular}




\begin{tabular}{l|llllllll}
$\delta=2$ & Min & $\ldots$ & FQUHAO & FQUGAO & FQUAO & FQUQAO & $\ldots$ & Max \\
$\ldots$ & $\ldots$ & $\ldots$ & $\ldots$ & $\ldots$ & $\ldots$ & $\ldots$ & $\ldots$ & $\ldots$ \\
$\delta=\infty$ & Min & $\ldots$ & Max & Max & Max & Max & $\ldots$ & Max \\
\hline
\end{tabular}

Abbreviations: $\mathrm{H}=$ harmonic; $\mathrm{G}=$ geometric and $\mathrm{Q}=$ quadratic. Note that here the FGUAO* means fuzzy geometric unified aggregation operator.

Observe that $\lambda$ and $\delta$ may have different values in the sub aggregations. Thus, an aggregation can have ten sub equations where two of them use a geometric structure, three a cubic aggregation, four an arithmetic average and finally, an harmonic one. Supposing that all of them follow the WA aggregation approach, the aggregation becomes the FWGA2WCA3WA4WHA. Following this methodology, we can analyse many different particular cases. For simplicity, let us summarize the study below focusing only on a general approach that uses generalized aggregation operators that include all the other aggregations.

For example, let us consider several aggregation operators with the same type of structure such as four fuzzy generalized WAs:

$F G U A O\left(a_{1}, \ldots, a_{n}\right)=C_{1} F G W A_{1}+C_{2} F G W A_{2}+C_{3} F G W A_{3}+C_{4} F G W A_{4}$.

Observe that the formula assigns a number to each FGWA in order to identify each of them. By doing so, we may analyse deeper structures of aggregations with seven, eight and more averages.

Similarly, we may consider fuzzy generalized probabilistic aggregations (FGPA). With four FGPAs, we obtain the following: $F G U A O\left(a_{1}, \ldots, a_{n}\right)=C_{1} F G P A_{1}+C_{2} F G P A_{2}+C_{3} F G P A_{3}+C_{4} F G P A_{4}$.

Similarly, we could also do the same for the FGOWA operator. For three OWA operators we get the following:

$F G U A O\left(a_{1}, \ldots, a_{n}\right)=C_{1} F G O W A_{1}+C_{2} F G O W A_{2}+C_{3} F G O W A_{3}$. 
Next, we are going to analyse more complex structures that use FGOWAs, FGWAs and FGPAs in the same aggregation. First, we shall consider the FGP3GOWA2GWA2 which is an aggregation technique that considers two GOWAs, three GWA, and two generalized probabilistic vectors:

$F G U A O\left(a_{1}, \ldots, a_{n}\right)=$

$=C_{1} F G P A_{1}+C_{2} F G P A_{2}+C_{3} F G P A_{3}+C_{3} F G O W A_{1}+C_{4} F G O W A_{2}+C_{5} F G W A_{1}+C_{6} F G W A_{2}$.

With this framework, we could consider deeper and more complex aggregations by using more sub aggregations in the system like the GP4GOWA5GWA4, the GP6GOWA9GWA8, and so on. Finally, it is possible to imagine or create extremely complicated situations where thousands of aggregations appear like a GP7000GOWA23000GWA16000. Observe that these approaches may appear frequently in the real world. Today, the problem in our real world is that we tend to simplify these difficulties and sometimes these simplified approaches may bring strong differences in the analysis leading us to different decisions. Note that other complex frameworks could be studied by using other types of aggregation operators such as heavy and induced aggregation operators, moving averages, and mixture operators [23].

Another interesting result appears when the FGPA, the FGWA or the FGOWA become the fuzzy generalized mean (FGM). Observe that this situation occurs when $w_{i}=1 / n$ for all $i$. Different scenarios also appear if some of the weighting vectors use the FGM while others do not. Therefore, we may build, for example, the FGPGOWAGWA2GM2:

$$
\begin{aligned}
& F G U A O\left(a_{1}, \ldots, a_{n}\right)= \\
& =C_{1} F G P A+C_{2} F G O W A+C_{3} F G W A_{1}+C_{4} F G W A_{2}+C_{5} F G M_{1}+C_{6} F G M_{2} .
\end{aligned}
$$


Other interesting approaches arise when we use the minimum and the maximum in the FGOWA aggregation because we establish the bounds assuming the data available in other aggregation operators. For example, we may build the FGPOWA2WA2MaxMin as follows: $F G U A O\left(a_{1}, \ldots, a_{n}\right)=$

$=C_{1} F G P A_{1}+C_{2} F G O W A_{1}+C_{3} F G O W A_{2}+C_{4} F G W A_{1}+C_{5} F G W A_{2}+C_{6} F M a x+C_{7} F M i n$.

As we can see, a lot of other potential semi boundary conditions can be constructed according to the aggregation operators used in the FGUAO operator. Moreover, we may consider other families of OWA and other aggregation operators, forming other types of structures based on the use of the centered-FGOWA, the olympic-FGOWA, the step-FGOWA and the median-FGOWA $[22,34,40]$.

Finally, let us look into an example when the FGUAO operator uses different types of arithmetic, quadratic and geometric aggregations. For example, we could construct an aggregation operator that combines the following ones: FPA, FOWA1, FOWA2, quadratic FOWA (FOWQA), geometric FOWA (FOWG), FWA, quadratic FWA (FWQA), harmonic FWA (FWHA), FA, quadratic FA (FQA), FMax and FMin. Thus, the aggregation operator formed can be represented as follows:

$\operatorname{FGUAO}\left(a_{1}, \ldots, a_{n}\right)=$

$\begin{aligned}= & C_{1} F P A+C_{2} F O W A_{1}+C_{3} F O W A_{2}+C_{4} F O W Q A+C_{5} F O W G+C_{6} F W A+ \\ & +C_{7} F W Q A+C_{8} F W H A+C_{9} F A+C_{10} F Q A+C_{11} F M a x+C_{12} F M i n\end{aligned}$.

Following this methodology, it is possible to develop a wide range of complex structures that combines a lot of different types of aggregation operators. Note that in simple analysis this type of structures may be considered too complex but it is important to remark that in real world problems where a lot of variables interact in the study, these type of 
aggregation structures seem to be some of the optimal ones that can correctly deal with the information.

\section{The quasi-FUAO operator}

The FGUAO operator can be further extended with quasi-arithmetic means forming the fuzzy quasi-arithmetic UAO (Quasi-FUAO) operator. It includes the FGUAO operator as a particular case and many other aggregations not included in the FGUAO by using a function in the aggregation process. Before introducing the Quasi-FUAO operator, let us briefly define the quasi-arithmetic mean [21] with fuzzy numbers (FQAM):

$\operatorname{FQAM}\left(\tilde{a}_{1}, \tilde{a}_{2}, \ldots, \tilde{a}_{n}\right)=g^{-1}\left(\frac{1}{n} \sum_{i=1}^{n} g\left(\tilde{a}_{i}\right)\right)$

where $g\left(\tilde{a}_{i}\right)$ is a strictly continuous monotonic function and $\tilde{a}_{i}$ are fuzzy numbers.

The Quasi-FUAO operator, which is a more general representation of the FQAM operator, can be defined as follows.

$\operatorname{QFUAO}\left(\tilde{a}_{1}, \tilde{a}_{2}, \ldots, \tilde{a}_{n}\right)=\sum_{h=1}^{m} C_{h} g_{h}^{-1}\left(\sum_{i=1}^{n} w_{i}^{h} g_{h}\left(\widetilde{a}_{i}^{\lambda}\right)\right)$

where $C_{h}$ is the degree of relevance that each sub aggregation has with $C_{h} \in[0,1]$ and $\sum_{h=1}^{m} C_{h}=1, w_{i}^{h}$ is the $i$ th weight of the $h$ th weighting vector $W$ with $w_{i}^{h} \in[0,1]$, $\sum_{i=1}^{n} w_{i}^{h}=1, g_{h}(\tilde{a})$ is a strictly continuous monotonic function and $\tilde{a}_{i}$ is the argument variable represented in the form of a fuzzy number.

Note that in this case we could also consider different functions $g(\tilde{a})$ for each sub aggregation. Moreover, we can also consider a second function $f(\tilde{a})$ for the weighting vector $C$. In this case, we get the following formulation: 
$\operatorname{QFUAO}\left(\tilde{a}_{1}, \tilde{a}_{2}, \ldots, \tilde{a}_{n}\right)=f_{h}^{-1}\left(\sum_{h=1}^{m} C_{h} f_{h}\left(g_{h}^{-1}\left(\sum_{i=1}^{n} w_{i}^{h} g_{h}\left(\widetilde{a}_{i}\right)\right)\right)\right)$,

where $f_{h}(\tilde{a})$ is a strictly continuous monotonic function.

With the Quasi-FUAO operator, it is also possible to consider a wide range of particular cases including the FUAO, the FUGAO, the FUQAO, the FGPA5, the FGWA4, the FGOWA6, the FGP4GOWA7GWA6 and many others. In Table 2, some of the main particular cases are presented.

Table 2

Families of Quasi-FUAO operators.

\begin{tabular}{lll}
\hline$g$ & $f$ & \multicolumn{1}{c}{ Aggregation operator } \\
\hline$g_{h}=a$ & $f=C_{h}$ & FUAO operator \\
$g_{h}=a^{2}$ & $f=C_{h}$ & FUQAO operator \\
$g_{h}=a^{-1}$ & $f=C_{h}$ & FUHAO operator \\
$g_{h}=a^{3}$ & $f=C_{h}$ & FUCAO operator \\
$g_{h} \rightarrow a^{0}$ & $f=C_{h}$ & FUGAO operator \\
$g_{h}=a^{2}$ & $f=C_{h}^{2}$ & FQUQAO operator \\
$g_{h}=a^{-1}$ & $f=C_{h}^{-1}$ & FHUHAO operator \\
$g_{h}=a^{3}$ & $f=C_{h}^{3}$ & FCUCAO operator \\
$g_{h} \rightarrow a^{0}$ & $f \rightarrow C_{h}^{0}$ & FGUGAO operator
\end{tabular}

Note that in Table 2 it has been assumed implicitly that $g_{1}=g_{2}=\ldots=g_{m}$ for all $h$. However, it is possible to consider situations where they are not equal.

\section{Multi-person decision making with the FGUAO operator}

The FGUAO and the Quasi-FUAO operators are generalized aggregation operators that could be implemented in multi-person decision-making problems [42]. Observe that the 
main advantage of a multi-person analysis is the provision of a deeper understanding of the framework because key decisions require the evaluation of many people. In this context, the main benefit of the FGUAO is that we can represent environments that deal with the attitudinal character of the decision maker, the objective and subjective data and considering the relevance of each aggregation technique.

The steps to follow with the FGUAO in a multi-person decision making process are: Step 1: Let $A=\left\{A_{1}, A_{2}, \ldots, A_{m}\right\}$ be a set of finite alternatives and $S=\left\{S_{1}, S_{2}, \ldots, S_{n}\right\}$, a set of finite states of nature, that form the matrix $\left(\tilde{a}_{g i}\right)_{m \times n}$. This matrix is created for each criteria $C R=\left(C r_{1}, C r_{2}, \ldots, C r_{x}\right)$. All these criterion are aggregated by using the weighting vector $Y=\left(y_{1}, y_{2}, \ldots, y_{t}\right)$ with $\sum_{e=1}^{t} y_{e}=1$ and $y_{e} \in[0,1]$.

Step 2: Let $E=\left\{E_{1}, E_{2}, \ldots, E_{q}\right\}$ be a finite set of decision makers. Let $Z=\left(z_{1}, z_{2}, \ldots, z_{q}\right)$ be the weighting vector of the decision makers such that $\sum_{k=1}^{q} z_{k}=1$ and $z_{k} \in[0,1]$. Each decision maker analyzes their own payoff matrix $\left(\tilde{a}_{g i}{ }^{(k)}\right)_{m \times n}$ after having considered the different available criteria. Use the fuzzy weighted average (FWA) to aggregate the information of the decision makers $E$ by using the weighting vector $Z=\left(z_{1}, z_{2}, \ldots, z_{q}\right)$. The result is the collective payoff matrix $\left(\tilde{a}_{g i}\right)_{m \times n}$. Thus, $x_{g i}=\sum_{k=1}^{q} z_{k} a_{g i}^{k}$.

Step 3: Obtain the weights of the FGPA, FGWA and FGOWA. Let us consider the following $h$ th weighting vector for the FGUAO: $W^{h}=\left(w_{1}^{h}, w_{2}^{h}, \ldots, w_{n}^{h}\right)$ such that $\sum_{i=1}^{n} w_{i}^{h}=1$ and $w_{i}^{h} \in[0,1]$.

Step 4: Calculate the aggregated results by using the FGUAO operator explained in Eq. (1) and (2). Analyze different families of FGUAO operators in order to obtain a better representation of the decision process. 
Step 5: Establish decisions following the results of the data studied in the previous steps. Select the strategy that give the most profitable result and form an ordering.

This decision framework uses the fuzzy multi-person - GUAO (MP-FGUAO) operator:

$\operatorname{MP-FGUAO}\left(\left(\tilde{a}_{1}{ }^{1}, \ldots, \tilde{a}_{1}{ }^{q}\right), \ldots,\left(\tilde{a}_{n}{ }^{1}, \ldots, \tilde{a}_{n}{ }^{q}\right)\right)=\sum_{h=1}^{m} C_{h}\left(\sum_{i=1}^{n} w_{i}^{h} \widetilde{a}_{i}^{\lambda_{h}}\right)^{1 / \lambda_{h}}$,

where $C_{h}$ is the relevance that each aggregation has with $C_{h} \in[0,1]$ and $\sum_{h=1}^{m} C_{h}=1$,

$\widetilde{a}_{i}=\sum_{k=1}^{q} z_{k} \widetilde{a}_{i}^{k}, \widetilde{a}_{i}^{k}$ is the argument variable provided by each person (or expert) after having considered the different criterion, $\widetilde{a}_{i}^{k}=\sum_{e=1}^{t} y_{e} \widetilde{a}_{i}^{e}, \widetilde{a}_{i}^{e}$ is the argument variable provided by each expert for each criteria represented in the form of a fuzzy number, $Y$ is a weighting vector of dimension $t$ with $\sum_{e=1}^{t} y_{e}=1$ and $y_{e} \in[0,1], \lambda_{h}$ is a parameter such that $\lambda_{h} \in(-\infty$, $\infty), Z$ is a weighting vector of dimension $q$ with $\sum_{k=1}^{q} z_{k}=1$ and $z_{k} \in[0,1]$ and there are $m$ weighting vectors $W$ of dimension $n$ with $\sum_{i=1}^{n} w_{i}^{h}=1$ and $w_{i}^{h} \in[0,1]$,

The MP-FGUAO obtains results connected to Section 3. For example, we can analyze the following operators:

- The multi-person - FGPOWAWA (MP-FGPOWAWA).

- The multi-person - FGP2OWA2WA2 (MP-FGP2OWA2WA2).

- The multi-person - FGP4OWA4WA4 (MP-FGP4OWA4WA4).

- The multi-person - FGP600OWA800WA900.

It is worth noting that more complex operators could be considered instead of using the FWA operator when aggregating the experts opinions. Among others, we may consider 
the FGUAO and its particular cases. Furthermore, it is also possible to consider situations where $C_{h}$ is not arithmetic as it is shown in Eq. (2).

\section{General applicability of the FGUAO operator}

The FGUAO and the Quasi-FUAO operators could be implemented in many areas including statistics, economics, computer science, engineering and management [13-14,17]. The main reason for this is that the FGUAO operators include all the particular classical aggregations as particular cases including the fuzzy average, the fuzzy weighted average and the FOWA operator. Thus, all the previous studies done with these classical aggregation operators could potentially be extended using this framework.

The work focuses on decision making $[8,45,51]$ at the European Union (EU). Note that the formation of the EU implies a lot of micro and macro decisions that should be taken in order to build a productive state. All the areas have to be considered including EU law, economics, politics, finance, marketing and management. Additionally, other decisions appear in many other areas where the EU should make a decision such as the Eurostat, the European Space Agency, chemistry, sports, arts and culture.

Let us look into an illustrative example in multi-person decision making in the European Monetary Union (EMU). Note that this type of problems is in the area of decision making in macroeconomics which usually deal with huge amounts of money or other equivalent variables. Therefore, it is critical to find the correct decisions because small deviations from the optimal result may produce huge economic differences in the region. It is also included two different criteria in the analysis so the example can be considered as a multi-criteria multi-person decision making problem. Note that this is very common in the EMU because the key decisions are usually influenced by several experts and criteria. 
Step 1: In this example, it is assumed that the EMU leaders are planning the general interest rate for the next year that the European Central Bank (ECB) should establish for the Eurozone. They consider the following five possible alternatives:

1. $A_{1}$ : Increase the interest rate $1 \%$.

2. $A_{2}$ : Increase the interest rate $0.5 \%$.

3. $A_{3}$ : Do not make any change.

4. $A_{4}$ : Decrease the interest rate $0.5 \%$.

5. $A_{5}$ : Decrease the interest rate $1 \%$.

After a deep study of the data, the experts of the EMU establish the following data regarding the alternatives. The assumption is that the potential benefits that these strategies may bring depend on two general criteria and five potential states of nature for each criterion:

1. $C r_{1}$ : Economic situation of the EMU (Regional - internal economic situation).
a. $S_{1}$ : Negative growth rate in the EMU.
b. $S_{2}$ : Growth rate close to 0 .
c. $S_{3}$ : Low positive growth rate.
d. $S_{4}$ : Positive growth rate.
e. $S_{5}$ : High growth rate.

2. $\mathrm{Cr}_{2}$ : World economic situation (External economic situation).
a. $S_{1}$ : Negative growth rate in the World.
b. $S_{2}$ : Growth rate close to 0 . 
c. $S_{3}$ : Low positive growth rate.

d. $S_{4}$ : Positive growth rate.

e. $S_{5}$ : High growth rate.

The group of experts of the EMU responsible for this decision is formed by 10 individuals divided in three general groups, each providing their own single opinion regarding the expected outcomes with each possible strategy. It is assumed that the first group has four experts and the other two groups, three experts. The results given by the first group are shown in Tables 3 and 4. Note that the results are valuations (numbers) between 0 and 100 .

Table 3

Expert 1 - Criteria 1 .

\begin{tabular}{llllll}
\hline & $S_{1}$ & $S_{2}$ & $S_{3}$ & $S_{4}$ & $S_{5}$ \\
\hline$A_{1}$ & $(30,40,50)$ & $(60,70,80)$ & $(50,60,70)$ & $(40,50,60)$ & $(80,90,100)$ \\
$A_{2}$ & $(60,70,80)$ & $(30,40,50)$ & $(80,90,100)$ & $(70,80,90)$ & $(40,50,60)$ \\
$A_{3}$ & $(70,80,90)$ & $(50,60,70)$ & $(30,40,50)$ & $(60,70,80)$ & $(70,80,90)$ \\
$A_{4}$ & $(30,40,50)$ & $(20,30,40)$ & $(40,50,60)$ & $(30,40,50)$ & $(30,40,50)$ \\
$A_{5}$ & $(40,50,60)$ & $(40,50,60)$ & $(60,70,80)$ & $(50,60,70)$ & $(50,60,70)$ \\
\hline
\end{tabular}

Table 4

Expert 1 - Criteria 2.

\begin{tabular}{llllll}
\hline & $S_{1}$ & $S_{2}$ & $S_{3}$ & $S_{4}$ & $S_{5}$ \\
\hline$A_{1}$ & $(50,60,70)$ & $(40,50,60)$ & $(30,40,50)$ & $(50,60,70)$ & $(40,50,60)$ \\
$A_{2}$ & $(70,80,90)$ & $(50,60,70)$ & $(40,50,60)$ & $(40,50,60)$ & $(50,60,70)$ \\
$A_{3}$ & $(30,40,50)$ & $(60,70,80)$ & $(70,80,90)$ & $(40,50,60)$ & $(30,40,50)$
\end{tabular}




$\begin{array}{llllll}A_{4} & (60,70,80) & (10,20,30) & (50,60,70) & (60,70,80) & (50,60,70) \\ A_{5} & (20,30,40) & (70,80,90) & (60,70,80) & (30,40,50) & (40,50,60)\end{array}$

With this information, the first group forms its general opinion. They assume that the first criteria have a degree of importance of $70 \%$ and the second one $30 \%$. The results are shown in Table 5.

Table 5

Expert $1-$ General expected result.

\begin{tabular}{llllll}
\hline & $S_{1}$ & $S_{2}$ & $S_{3}$ & $S_{4}$ & $S_{5}$ \\
\hline$A_{1}$ & $(36,46,56)$ & $(54,64,74)$ & $(44,54,64)$ & $(43,53,63)$ & $(68,78,88)$ \\
$A_{2}$ & $(53,63,73)$ & $(36,46,56)$ & $(68,78,88)$ & $(61,71,81)$ & $(43,53,63)$ \\
$A_{3}$ & $(58,68,78)$ & $(53,63,73)$ & $(42,52,62)$ & $(54,64,74)$ & $(58,68,78)$ \\
$A_{4}$ & $(39,49,59)$ & $(17,27,37)$ & $(43,53,63)$ & $(39,49,59)$ & $(36,46,56)$ \\
$A_{5}$ & $(34,44,54)$ & $(49,59,69)$ & $(60,70,80)$ & $(44,54,64)$ & $(47,57,67)$ \\
\hline
\end{tabular}

A similar methodology is used for the other two groups of experts. The second group also considers that the first criterion has a relevance of $70 \%$ while the second one has $30 \%$. Table 6 and 7 presents the expected results for each criteria and Table 8 the general expected result of the second group.

Table 6 
Expert 2-Criteria 1.

\begin{tabular}{llllll}
\hline & $S_{1}$ & $S_{2}$ & $S_{3}$ & $S_{4}$ & $S_{5}$ \\
\hline$A_{1}$ & $(30,40,50)$ & $(20,30,40)$ & $(70,80,90)$ & $(30,40,50)$ & $(60,70,80)$ \\
$A_{2}$ & $(20,30,40)$ & $(40,50,60)$ & $(40,50,60)$ & $(50,60,70)$ & $(50,60,70)$ \\
$A_{3}$ & $(10,20,30)$ & $(50,60,70)$ & $(30,40,50)$ & $(40,50,60)$ & $(60,70,80)$ \\
$A_{4}$ & $(60,70,80)$ & $(30,40,50)$ & $(60,70,80)$ & $(60,70,80)$ & $(30,40,50)$ \\
$A_{5}$ & $(70,80,90)$ & $(60,70,80)$ & $(70,80,90)$ & $(50,60,70)$ & $(20,30,40)$ \\
\hline
\end{tabular}

Table 7

Expert 2 - Criteria 2.

\begin{tabular}{llllll}
\hline & $S_{1}$ & $S_{2}$ & $S_{3}$ & $S_{4}$ & $S_{5}$ \\
\hline$A_{1}$ & $(40,50,60)$ & $(60,70,80)$ & $(50,60,70)$ & $(30,40,50)$ & $(20,30,40)$ \\
$A_{2}$ & $(30,40,50)$ & $(20,30,40)$ & $(20,30,40)$ & $(50,60,70)$ & $(40,50,60)$ \\
$A_{3}$ & $(70,80,90)$ & $(30,40,50)$ & $(10,20,30)$ & $(60,70,80)$ & $(40,50,60)$ \\
$A_{4}$ & $(40,50,60)$ & $(50,60,70)$ & $(60,70,80)$ & $(40,50,60)$ & $(50,60,70)$ \\
$A_{5}$ & $(70,80,90)$ & $(60,70,80)$ & $(30,40,50)$ & $(30,40,50)$ & $(40,50,60)$ \\
\hline
\end{tabular}

Table 8

Expert 2 - General expected result.

\begin{tabular}{llllll}
\hline & $S_{1}$ & $S_{2}$ & $S_{3}$ & $S_{4}$ & $S_{5}$ \\
\hline$A_{1}$ & $(33,43,53)$ & $(32,42,52)$ & $(64,74,84)$ & $(30,40,50)$ & $(48,58,68)$ \\
$A_{2}$ & $(23,33,43)$ & $(34,44,54)$ & $(34,44,54)$ & $(50,60,70)$ & $(47,57,67)$ \\
$A_{3}$ & $(28,38,48)$ & $(44,54,64)$ & $(24,34,44)$ & $(46,56,66)$ & $(54,64,74)$
\end{tabular}




$\begin{array}{llllll}A_{4} & (54,64,74) & (36,46,56) & (60,70,80) & (54,64,74) & (36,46,56) \\ A_{5} & (70,80,90) & (60,70,80) & (58,68,78) & (44,54,64) & (26,36,46)\end{array}$

The third group assumes that the first criteria are more relevant giving a degree of $80 \%$ and a degree of $20 \%$ for the second one. Table 9 and 10 shows their opinions for each criteria and Table 11 their general expected result.

Table 9

Expert 3 - Criteria 1.

\begin{tabular}{llllll}
\hline & $S_{1}$ & $S_{2}$ & $S_{3}$ & $S_{4}$ & $S_{5}$ \\
\hline$A_{1}$ & $(30,40,50)$ & $(50,60,70)$ & $(60,70,80)$ & $(30,40,50)$ & $(40,50,60)$ \\
$A_{2}$ & $(40,50,60)$ & $(60,70,80)$ & $(30,40,50)$ & $(40,50,60)$ & $(50,60,70)$ \\
$A_{3}$ & $(50,60,70)$ & $(70,80,90)$ & $(50,60,70)$ & $(40,50,60)$ & $(60,70,80)$ \\
$A_{4}$ & $(60,70,80)$ & $(30,40,50)$ & $(40,50,60)$ & $(50,60,70)$ & $(40,50,60)$ \\
$A_{5}$ & $(20,30,40)$ & $(40,50,60)$ & $(60,70,80)$ & $(70,80,90)$ & $(30,40,50)$ \\
\hline
\end{tabular}

Table 10

Expert 3 - Criteria 2.

\begin{tabular}{llllll}
\hline & $S_{1}$ & $S_{2}$ & $S_{3}$ & $S_{4}$ & $S_{5}$ \\
\hline$A_{1}$ & $(40,50,60)$ & $(70,80,90)$ & $(50,60,70)$ & $(50,60,70)$ & $(30,40,50)$ \\
$A_{2}$ & $(50,60,70)$ & $(60,70,80)$ & $(40,50,60)$ & $(30,40,50)$ & $(30,40,50)$ \\
$A_{3}$ & $(30,40,50)$ & $(20,30,40)$ & $(40,50,60)$ & $(60,70,80)$ & $(50,60,70)$
\end{tabular}




$\begin{array}{llllll}A_{4} & (60,70,80) & (40,50,60) & (50,60,70) & (40,50,60) & (40,50,60) \\ A_{5} & (40,50,60) & (50,60,70) & (30,40,50) & (60,70,80) & (40,50,60)\end{array}$

Table 11

Expert 3 - General expected result.

\begin{tabular}{llllll}
\hline & $S_{1}$ & $S_{2}$ & $S_{3}$ & $S_{4}$ & $S_{5}$ \\
\hline$A_{1}$ & $(32,42,52)$ & $(54,64,74)$ & $(58,68,78)$ & $(34,44,54)$ & $(38,48,58)$ \\
$A_{2}$ & $(42,52,62)$ & $(60,70,80)$ & $(32,42,52)$ & $(38,48,58)$ & $(46,56,66)$ \\
$A_{3}$ & $(46,56,66)$ & $(60,70,80)$ & $(48,58,68)$ & $(44,54,64)$ & $(58,68,78)$ \\
$A_{4}$ & $(60,70,80)$ & $(32,42,52)$ & $(42,52,62)$ & $(48,58,68)$ & $(40,50,60)$ \\
$A_{5}$ & $(24,34,44)$ & $(42,52,62)$ & $(54,64,74)$ & $(68,78,88)$ & $(32,42,52)$ \\
\hline
\end{tabular}

Step 2: With the data of the previous tables, we aggregate it in order to form a collective results. This study assumes that the first group has more importance than the other two groups. Thus, the weighting vector that represents the importance of the experts is: $X=$ $(0.4,0.3,0.3)$. The results are shown in Table 12.

Table 12 
Collective results - General expected result.

\begin{tabular}{lllllll}
\hline & $S_{1}$ & $S_{2}$ & $S_{3}$ & $S_{4}$ & $S_{5}$ \\
\hline$A_{1}$ & $(33.9,43.9,53.9)$ & $(47.4,57.4,67.4)$ & $(54.2,64.2,74.2)$ & $(36.4,46.4,56.4)$ & $(53,63,73)$ \\
$A_{2}$ & $(40.7,50.7,60.7)$ & $(42.6,52.6,62.6)$ & $(47,57,67)$ & $(50.8,60.8,70.8)$ & $(45.1,55.1,65.1)$ \\
$A_{3}$ & $(45.4,55.4,65.4)$ & $(52.4,62.4,72.4)$ & $(38.4,48.4,58.4)$ & $(48.6,58.6,68.6)$ & $(56.8,66.8,76.8)$ \\
$A_{4}$ & $(49.8,59.8,69.8)$ & $(27.2,37.2,47.2)$ & $(47.8,57.8,67.8)$ & $(46.2,56.2,66.2)$ & $(37.2,47.2,57.2)$ \\
$A_{5}$ & $(41.8,51.8,61.8)$ & $(50.2,60.2,70.2)$ & $(57.6,67.6,77.6)$ & $(51.2,61.2,71.2)$ & $(36.2,46.2,56.2)$
\end{tabular}

Step 3: Next, aggregate the information to select an alternative. Several methods are considered from the minimum to the maximum. For the weighted average, let us assume that $V=(0.3,0.2,0.2,0.2,0.1)$; for the probabilistic information $P=(0.3,0.3,0.2,0.1,0.1)$ and for the OWA: $W_{1}=(0.1,0.2,0.2,0.2,0.3)$.

Step 4: In this example let us consider the fuzzy average, fuzzy minimum, fuzzy maximum, fuzzy probabilistic aggregation, fuzzy weighted average, fuzzy OWA and FPOWA2WA, being the weighting vector of the second OWA as follows: $W_{2}=(0.1,0.1,0.2$, $0.3,0.3)$. Note that the importance of the coefficients $C$ are: $C=(0.3,0.1,0.2,0.4)$. Table 13 presents the results.

Table 13

Aggregated results.

\begin{tabular}{lllllll}
\hline \multicolumn{2}{c}{ FMin } & FMax & FWA & FPA & FOWA & FPOWA2WA \\
\hline$A_{1}$ & $(33.9,43.9,53.9)$ & $(54.2,64.2,74.2)$ & $(43.0,53.0,63.0)$ & $(44.1,54.1,64.1)$ & $(42.9,52.9,62.9)$ & $(42.96,52.96,62.96)$ \\
$A_{2}$ & $(40.7,50.7,60.7)$ & $(50.8,60.8,70.8)$ & $(44.8,54.8,64.8)$ & $(43.9,53.9,63.9)$ & $(44.2,54.2,64.2)$ & $(44.25,54.25,64.25)$ \\
$A_{3}$ & $(38.4,48.4,58.4)$ & $(56.8,66.8,76.8)$ & $(47.1,57.1,67.1)$ & $(47.5,57.5,67.5)$ & $(46.4,56.4,66.4)$ & $(46.87,56.87,66.87)$
\end{tabular}



$A_{4} \quad(27.2,37.2,47.2) \quad(49.8,59.8,69.8) \quad(42.9,52.9,62.9) \quad(41,51,61)$
$(39.3,49.3,59.3) \quad(41.03,51.03,61.03)$
$A_{5} \quad(36.2,46.2,56.2) \quad(57.6,67.6,77.6)$
$(47.9,57.9,67.9)$
$(47.8,57.8,67.8)$
$(45.2,55.2,65.2) \quad(46.88,56.88,66.88)$

In order to deepen into the results and compare the alternatives, let us presents the results graphically for the central value in each alternative. Thus, we can see the results and the differences between them. Figure 1 presents the results.

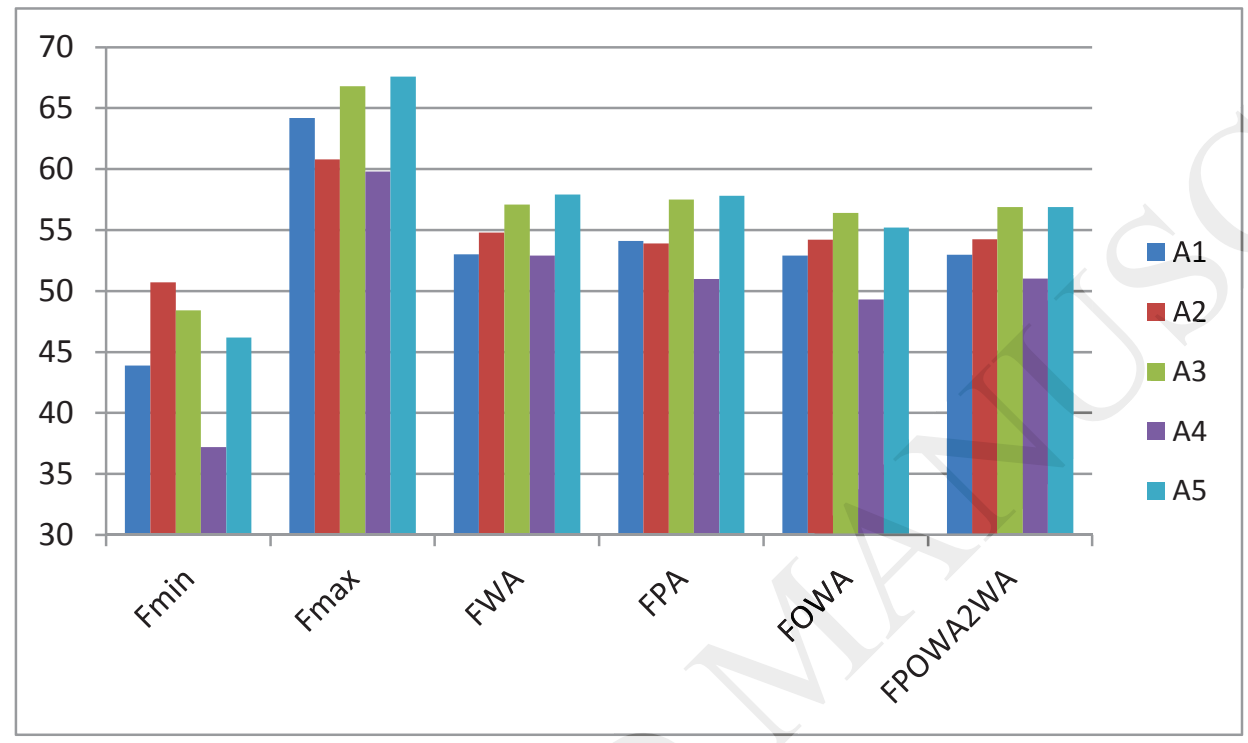

Figure 1. Graphical visualization of the aggregated results.

Step 5: According to this information, the optimal choice is $A_{5}$, although for the FOWA operator the optimal one is $A_{3}$ and for the minimum, $A_{2}$. In order to represent this information in a more complete way, in Table 14 it is presented the ranking of the alternatives for each type of aggregation operator considered in the previous Table.

\section{Table 14}

Ranking of the alternatives.

\begin{tabular}{|l|l|l|l|}
\hline & Ranking & & Ranking \\
\hline
\end{tabular}




\begin{tabular}{|l|l|l|l|}
\hline FMin & $\left.\left.\left.\left.A_{2}\right\} A_{3}\right\} A_{5}\right\} A_{1}\right\} A_{4}$ & FWA & $\left.\left.\left.\left.A_{5}\right\} A_{3}\right\} A_{1}\right\} A_{2}\right\} A_{4}$ \\
\hline FMax & $\left.\left.\left.\left.A_{5}\right\} A_{3}\right\} A_{1}\right\} A_{2}\right\} A_{4}$ & FOWA & $\left.\left.\left.\left.A_{3}\right\} A_{5}\right\} A_{2}\right\} A_{1}\right\} A_{4}$ \\
\hline FPA & $\left.\left.\left.\left.A_{5}\right\} A_{3}\right\} A_{2}\right\} A_{1}\right\} A_{4}$ & FPOWA2WA & $\left.\left.\left.\left.A_{5}\right\} A_{3}\right\} A_{2}\right\} A_{1}\right\} A_{4}$ \\
\hline
\end{tabular}

The optimal choice is $A_{5}$. But depending on the particular type of aggregation operator used, the results may lead to different decisions. Obviously, each decision maker will select the alternative in closest accordance to his interests. Thus, in this example, the decision consists in selecting the optimal alternative found when using the FPOWA2WA operator, that is, $A_{5}$.

\section{Conclusions}

The FGUAO operator has been introduced. It provides a unified framework where we can to analyze many different structures of fuzzy aggregation operators and a wide range of unifications between them. We see that it includes the classical fuzzy aggregations including the FAM, the FWA, the FOWA, the FGWA, the FGOWA and many others as particular cases. Thus, it represents a further generalization of the previous approaches by providing a more general structure that can adapt to the complex environments in a more efficient and flexible way. Moreover, it can include complex aggregation operators that deal with complex environments such as problems with a wide range of sub-aggregations such as a FGP2OWA3WA2 operator or a FGP15OWA24WA29 operator. The work consider many particular cases based on the notion of generalized aggregation forming arithmetic, geometric and quadratic aggregations such as the fuzzy UAO (FUAO), the fuzzy unified geometric aggregation operator (FUGAO) and the fuzzy unified quadratic aggregation operator (FUQAO). We have further generalized the FGUAO by using quasi-arithmetic means forming the Quasi-FUAO. 
The article also studies the applicability of the FGUAO operator and we see that it is very broad because previous works that use any aggregation operator included in the FGUAO operator could potentially be revised with this new framework. Thus, obtaining a more complete picture of the problem that can always be reduced to the classical approach if necessary. We have focused on multi-criteria multi-person decision making problems forming a new aggregation operator that we call the MP-FGUAO operator. Its key advantage is that it permits to deal with the opinion of several experts in the analysis. We have developed an illustrative example in a European decision making problem regarding the selection of the optimal interest rate for the EMU. The main advantage of the FGUAO operator is that it can deal with this type of problems in an efficient way because it is a flexible aggregation operator that can adapt easily to different situations where each country may provide different opinions. Note that these types of problems are in the area of decision making in macroeconomics which usually involve huge quantities of money. Therefore, finding the correct decision is critical in order to maximize the benefits or minimize the costs as much as possible.

In future research, we shall introduce further generalizations of this framework by using other characteristics in the formulation including Bonferroni means [4,52], induced aggregation operators [22] and norms [1]. We also expect to study more applications in other areas such as in engineering, economics and statistics [2] giving special emphasis to decisions $[11,50]$ with a particular focus at European and World level.

\section{Acknowledgements}

We would like to thank the associate editor and the anonymous reviewers for valuable comments that have improved the quality of the paper. Support from the Chilean Government 
through the Fondecyt Regular program (project number 1160286), the University of Chile and from the project PIEF-GA-2011-300062 of the European Commission, is gratefully acknowledged. 


\section{References}

[1] G. Beliakov, A. Pradera, T. Calvo, Aggregation functions: A guide for practitioners, Springer-Verlag, Berlin, 2007.

[2] J. Belles-Sampera, J.M. Merigó, M. Guillén, M. Santolino, The connection between distortion risk measures and ordered weighted averaging operators, Insurance: Mathematics and Economics 52 (2013) 411-420.

[3] F. Blanco-Mesa, J.M. Merigó, A.M. Gil-Lafuente, Fuzzy decision making: A bibliometric-based review, Journal of Intelligent \& Fuzzy Systems 32 (2017) 20332050.

[4] F. Blanco-Mesa, J.M. Merigó, J. Kacprzyk, Bonferroni means with distance measures and the adequacy coefficient in entrepreneurial group theory, Knowledge-Based Systems 111 (2016) 217-227.

[5] S.J. Chen, S.M. Chen, A new method for handling multi-criteria fuzzy decision making problems using FN-IOWA operators, Cybernetics \& Systems 34 (2003) 109137.

[6] D. Dubois, H. Prade, Fuzzy sets and systems: Theory and applications, Academic Press, New York, 1980.

[7] K.J. Engemann, D.P. Filev, R.R. Yager, Modelling decision making using immediate probabilities, International Journal of General Systems 24 (1996) 281-294.

[8] J. Figueira, S. Greco, M. Ehrgott, Multiple criteria decision analysis: State of the art surveys, Springer, Boston, 2005.

[9] J. Fodor, J.L. Marichal, M. Roubens, Characterization of the ordered weighted averaging operators, IEEE Transactions on Fuzzy Systems 3 (1995) 236-240. 
[10] M. Grabisch, J.L. Marichal, R. Mesiar, E. Pap, Aggregation functions: Means, Information Sciences 181 (2011) 1-22.

[11] Y.D. He, H.Y. Chen, L.G. Zhou, J.P. Liu, Z.F. Tao, Intuitionistic fuzzy geometric interaction averaging operators and their application to multi-criteria decision making, Information Sciences 259 (2014) 142-159.

[12] A. Kaufmann, M.M. Gupta, Introduction to fuzzy arithmetic, Publications Van Nostrand, Rheinhold, 1985.

[13] S. Laengle, G. Loyola, J.M. Merigó, Mean-variance portfolio selection with the ordered weighted average, IEEE Transactions on Fuzzy Systems 25 (2017) 350-362.

[14] E. León-Castro, E. Avilés-Ochoa, J.M. Merigó, A.M. Gil-Lafuente, Heavy moving averages and their application in econometric forecasting, Cybernetics \& Systems 49 (2018) 26-43.

[15] J.P. Liu, S. Lin, H.Y. Chen, Q. Xu, Penalty-based continuous aggregation operators and their application to group decision making, Knowledge-Based Systems 37 (2013) $490-501$.

[16] P.D. Liu, J.L. Liu, J.M. Merigó, Partitioned Heronian means based on linguistic intuitionistic fuzzy numbers for dealing with multi-attribute group decision making, Applied Soft Computing 62 (2018) 395-422.

[17] S. Maldonado, J.M. Merigó, J. Miranda, Redefining support vector machines with the ordered weighted average, Knowledge-Based Systems 148 (2018) 41-46.

[18] J.M. Merigó, Fuzzy decision making using immediate probabilities, Computers \& Industrial Engineering 58 (2010) 651-657.

[19] J.M. Merigó, Fuzzy multi-person decision making with fuzzy probabilistic aggregation operators, International Journal of Fuzzy Systems, 13 (2011) 163-174. 
[20] J.M. Merigó. The probabilistic weighted average and its application in multi-person decision making, International Journal of Intelligent Systems, 27 (2012) 457-476.

[21] J.M. Merigó, M. Casanovas, The fuzzy generalized OWA operator and its application in strategic decision making, Cybernetics \& Systems 41 (2010) 359-370.

[22] J.M. Merigó, A.M. Gil-Lafuente, The induced generalized OWA operator, Information Sciences 179 (2009) 729-741.

[23] J.M. Merigó, A.M. Gil-Lafuente, The induced 2-tuple linguistic generalized OWA operator and its application in linguistic decision making, Information Sciences 236 (2013) 1-16.

[24] J.M. Merigó, A.M. Gil-Lafuente, R.R. Yager, An overview of fuzzy research with bibliometric indicators, Applied Soft Computing 27 (2015) 420-433.

[25] J.M. Merigó, C. Lobato-Carral, A Carrilero-Castillo, Decision making in the European Union under risk and uncertainty, European Journal of International Management, 6 (2012) 590-609.

[26] C.E. Shannon, A mathematical theory of communication, Bell System Technical Journal 27 (1948) 379-423, 623-656.

[27] S. Roychowdhury, W. Pedrycz, A survey of defuzzification strategies, International Journal of Intelligent Systems 16 (2001) 679-695.

[28] V. Torra, The weighted OWA operator, International Journal of Intelligent Systems 12 (1997) 153-166.

[29] Y.M. Wang, Y. Luo, Generalised fuzzy weighted mean and its applications, International Journal of General Systems 38 (2009) 533-546. 
[30] G.W. Wei, R. Lin, X. Zhao, H.J. Wang, An approach to multiple attribute decision making based on the induced Choquet integral with fuzzy number intuitionistic fuzzy information, Journal of Business Economics and Management 15 (2014) 277-298.

[31] Z.S. Xu, Fuzzy harmonic mean operators, International Journal of Intelligent Systems 24 (2009) 152-172.

[32] Z.S. Xu, Q.L. Da, An overview of operators for aggregating information, International Journal of Intelligent Systems 18 (2003) 953-969.

[33] R.R. Yager, On ordered weighted averaging aggregation operators in multi-criteria decision making, IEEE Transactions on Systems, Man and Cybernetics B 18 (1988) 183-190.

[34] R.R. Yager, Families of OWA operators, Fuzzy Sets and Systems 59 (1993) 125-148.

[35] R.R. Yager, Including importances in OWA aggregation using fuzzy systems modelling, IEEE Transactions on Fuzzy Systems 6 (1998) 286-294.

[36] R.R. Yager, New modes of OWA information fusion, International Journal of Intelligent Systems 13 (1998) 661-681.

[37] R.R. Yager, Generalized OWA aggregation operators, Fuzzy Optimization and Decision Making 3 (2004) 93-107.

[38] R.R. Yager, K.J. Engemann, D.P. Filev, On the concept of immediate probabilities, International Journal of Intelligent Systems 10 (1995) 373-397.

[39] R.R. Yager, J. Kacprzyk, The ordered weighted averaging operators: Theory and applications, Kluwer Academic Publishers, Norwell, MA, 1997.

[40] R.R. Yager, J. Kacprzyk, G. Beliakov, Recent developments on the ordered weighted averaging operators: Theory and practice, Springer-Verlag, Berlin, 2011. 
[41] D. Yu, D.F. Li, J.M. Merigó, L. Fang, Mapping development of linguistic decision making studies, Journal of Intelligent \& Fuzzy Systems 30(5) (2016) 2727-2736.

[42] B. Yusoff, J.M. Merigó, D. Ceballos, OWA-based aggregation methods in multiexpert MCDM model, Economic Computation and Economic Cybernetics Studies and Research 51(2) (2017) 211-230.

[43] L.A. Zadeh, Fuzzy sets, Information and Control 8 (1965) 338-353.

[44] L.A. Zadeh, The concept of a linguistic variable and its application to approximate reasoning. Part 1, Information Sciences 8 (1975) 199-249; Part 2, Information Sciences 8 (1975) 301-357; Part 3, Information Sciences 9 (1975) 43-80.

[45] E.K. Zavadskas, Z. Turskis, Multiple criteria decision making (MCDM) methods: An overview, Technological and Economic Development of Economy 17 (2011) 397-427.

[46] S.Z. Zeng, W. Su, Intuitionistic fuzzy ordered weighted distance operator, KnowledgeBased Systems 24 (2011) 1224-1232.

[47] S.Z. Zeng, W. Su, A. Le, Fuzzy generalized ordered weighted averaging distance operator and its application to decision making, International Journal of Fuzzy Systems 14 (2012) 402-412.

[48] H. Zhao, Z.S. Xu, M. Ni, S. Liu, Generalized aggregation operators for intuitionistic fuzzy sets, International Journal of Intelligent Systems 24 (2010) 1-30.

[49] L.G. Zhou, H.Y. Chen, J.P. Liu, Generalized power aggregation operators and their applications in group decision making, Computers \& Industrial Engineering 62 (2012) 989-999.

[50] L.G. Zhou, Z.F. Tao, H.Y. Chen, J.P. Liu, Continuous interval-valued intuitionistic fuzzy aggregation operators and their applications to group decision making, Applied Mathematical Modelling 38 (2014) 2190-2205. 
[51] L.G. Zhou, J.X. Wu, H.Y. Chen, Linguistic continuous ordered weighted distance measure and its application to multiple attributes group decision making, Applied Soft Computing 25 (2014) 266-276.

[52] B. Zhu, Z.S. Xu, M.M. Xia, Hesitant fuzzy geometric Bonferroni means, Information Sciences 205 (2012) 72-85. 